\title{
Comportement du sol au labour : méthode d'analyse et évaluation des conséquences de l'état initial du sol sur l'état transformé par le labour
}

\author{
I Coulomb 1, J Caneill 2, H Manichon 3 \\ 1 INRA, agronomie, 78850 Thiverval-Grignon \\ 2 INA-PG, 16, rue Claude-Bernard, 75231 Paris Cedex 05; \\ ${ }^{3}$ CIRAD, BP 5035, 34032 Montpellier, France
}

(Reçu le 1er juin 1992; accepté le 2 novembre 1992)

\begin{abstract}
Résumé - Nous étudions expérimentalement l'effet de l'état structural de la couche labourée avant labour sur son état après labour, en sol limono-argileux dans le domaine hydrique de comportement fragile du sol. Nous comparons deux traitements caractérisés par des états avant labour continus et compacts induits par compactage ou fragmentaires. On distingue deux types d'états compactés en fonction de la date de tassement (1 semaine avant labour, 1 mois avant labour). Trois classes de vitesses de labour (de 1 à $8 \mathrm{~km} / \mathrm{h}$ ) sont explorées. Après avoir présenté la méthode, nous montrons que les actions expérimentales avant labour ont effectivement engendré des états structuraux contrastés et structurés selon les traitements. Nous analysons alors la persistance après labour de différences entre les états. La caractérisation de l'état structural est fondée sur une observation morphologique quantifiée du profil cultural. La variabilité spatiale des états après labour est analysée sur la base d'analyses statistiques descriptives des données. Elle se structure selon les traitements, ce qui confirme l'effet déterminant de l'état initial du sol sur l'état transformé par le labour. La vitesse de labour a peu d'effet. Pour un état initialement fragmentaire, l'état après labour est semblable à l'état initial, avec des mottes de taille légèrement inférieure et un volume apparent plus important; pour un état continu et compact, l'état final se caractérise par des vides importants et des blocs décimétriques fortement tassés, de taille variable selon l'ancienneté du compactage.
\end{abstract}

labour / état structural / mottes / porosité / profil cultural

Summary - Behaviour of soil during ploughing: method and estimation of the consequences of soil structure before ploughing for soil structure after ploughing. The effect of soil structure in the ploughed layer before ploughing was studied in field experiments, in clay loam soils with a friable behaviour. Two treatments, leading to a soil structure with small and non-adherent clods or to a massive and compacted ploughed layer before ploughing were compared. Two types of compacted soil structure were distinguished according to the date of compaction (1 wk before ploughing, 1 month before ploughing). Three tractor speeds ranging from $1-8 \mathrm{~km} / \mathrm{h}$ were investigated. After presentation of the method, soil structure before ploughing was characterized according to the treatments, and the persistence of differences after ploughing was analyzed. Soil structure was characterized by a quantifiable morphological description based on mapping of the ploughed layer profile. Soil structure spatial variability after ploughing was described with statistical data analysis. It was linked to the treatments. This results show the crucial effect of initial soil structure on soil structure after ploughing. No speed effect appeared. When soil structure before ploughing was made up of small and distinctive clods, soil structure after ploughing was almost the same as initial structure with a lower density; when soil structure before ploughing was massive and compacted, the ploughed layer was characterized by decimetrical clods. Their size depended on the date of compaction.

ploughing / soil structure / clod / porosity / soil profile 


\section{INTRODUCTION}

Le labour, avec une charrue à socs et versoirs, est couramment pratiqué chaque année dans les parcelles de grande culture. II se justifie, selon les cas, par ses effets sur la destruction des plantes adventices, sur l'incorporation de matières organiques ou d'engrais au sol, sur la dilution des pesticides phytotoxiques, ou enfin, sur l'état du profil cultural et l'amélioration de la circulation de l'eau saturante.

C'est une opération culturale importante au sein de l'itinéraire technique, en raison des coûts qu'elle induit et des conséquences qu'elle entraîne sur la mise en place et le fonctionnement du peuplement végétal et de son système racinaire. Elle est importante aussi par le volume de sol concerné, l'état du sol ne pouvant plus dans la pratique être corrigé en profondeur avant la prochaine opération de même nature.

L'observation des champs cultivés montre qu'il existe de grandes différences entre les états du sol obtenus après labour, sans que cela corresponde à des objectifs recherchés par les praticiens (Hénin et al, 1969; Manichon et Sebillotte, 1975). Ces différences s'expriment par le degré de fragmentation plus ou moins intense de la couche labourée, par son volume apparent, par le calibre et l'état interne des mottes (espace poral, résistance mécanique) (Manichon, 1982a et b). Cette variabilité se retrouve aussi au sein des parcelles (Manichon, 1988). Cela suggère l'influence de deux facteurs : la résistance mécanique du sol au moment du travail et la contrainte mécanique appliquée par l'outil.

Dans la pratique, c'est la vitesse d'avancement qui est susceptible d'entraîner des variations de résultats dues au second facteur pour un outil et une profondeur de travail déterminés.

La variabilité du milieu explique, en partie, les variations du premier facteur: I'influence de la texture et de l'humidité du sol (Atterberg, 1911; Nichols et Baver, 1930; Faure, 1971; Rémy, 1971; Guérif, 1990) est connue. Mais I'on ne peut invoquer ces causes pour expliquer la variabilité des états du sol constatée lors d'enquêtes agronomiques entre parcelles d'un même type de sol, travaillées dans les mêmes conditions d'humidité (Coulomb et al, 1990; Manichon et al, 1988). L'état structural de la couche de sol au moment du labour, qui dépend de l'histoire culturale de la parcelle, et en particulier des compactages subis antérieurement au labour, a été mis en cause dans les travaux cités et dans ceux de Papy (1984). Certains travaux en laboratoire viennent à l'appui de cette hypothèse : la résistance mécanique en traction augmente avec la masse volumique du sol (Monnier et Guérif, 1988); des travaux en milieux pulvérulents désordonnés montreraient un effet de la taille et la forme des éléments structuraux (Dantu, 1957), de la proportion de grains mous et durs (Travers et al, 1988) sur la transmission des contraintes; de plus, les conditions de compactage (en particulier la teneur en eau) pour atteindre une masse volumique du sol donnée (Guérif, 1988, 1990) modifient la résistance mécanique en traction du sol; enfin, par un effet de thixotropie, les résistances mécaniques à la pénétration, à la rupture et à la compression augmenteraient avec le temps (age-hardening) sans qu'il y ait modification de la densité ou de la teneur en eau, à la suite d'un réarrangement entre les particules d'argile (Utomo et Dexter, 1981; Dexter, 1988). Notons que ces travaux de laboratoire sont réalisés sur des volumes de matériau de l'ordre du $\mathrm{mm} 3$ ou dm3. L'extrapolation à un volume de couche labourée pose des problèmes méthodologiques mal résolus.

L'existence d'une interaction entre l'état structural initial du sol et l'état transformé par le labour est donc vraisemblable. Mais les résultats d'enquêtes au champ, qui sont à l'origine de cette hypothèse, sont insuffisants pour la démontrer. Leur interprétation repose en effet sur la mise en correspondance d'états après labour, avec des interventions culturales classées selon la dégradation potentielle qu'elles peuvent induire avant labour. L'état avant labour n'est en fait jamais caractérisé. De plus, chaque parcelle étudiée est associée à une combinaison donnée des facteurs énoncés, qu'il n'est pas possible de séparer.

Une approche expérimentale est nécessaire pour séparer ces facteurs et analyser leur rôle. Elle consiste, après avoir créé dans une parcelle une gamme d'états structuraux représentative de ceux que l'on trouve couramment au champ, à labourer celle-ci dans des conditions d'humidité favorables à la fragmentation du sol (consistance fragile) avec une même charrue. Les états du sol avant labour étant connus, l'existence et la persistance de différences entre les états obtenus après des labours effectués à différentes vitesses peuvent être étudiées. Cela suppose l'acquisition d'une information sur la variabilité spatiale des états avant et après labour qui puisse être reliée aux passages de la charrue, ceux-ci étant définis par des paramètres de fonctionne- 
ment de l'outil. La caractérisation de l'état structural du sol doit permettre de distinguer l'effet du labour sur la structure des mottes et de leurs assemblages, de l'effet du climat sur cette structure. Pour comparer l'ampleur de la fragmentation réalisée par la charrue dans différentes conditions, la détermination de variables quantitatives est recherchée. La méthode du profil cultural (Manichon, 1982a), dont l'objectif est de décrire la structure des couches travaillées de façon à observer et analyser l'effet des systèmes de culture et des itinéraires techniques sur le sol, est susceptible de répondre à ces exigences méthodologiques. Elle permet en effet de décrire séparément les volumes ayant subi des contraintes mécaniques différentes et de retenir des critères morphologiques supposés être directement reliés à une contrainte identifiée par sa nature , son intensité et ses conditions d'application. Elle présente par ce fait le gros avantage, par rapport à une analyse globale de la porosité, de relier structure du sol et opération culturale (Stengel, 1990). Elle servira de base à la méthode de caractérisation développée dans le présent article, avec un souci particulier de quantifier certains critères de description. Dans cet article, nous présentons la méthode utilisée et l'analyse morphologique des états structuraux avant et après labour en fonction des actions expérimentales effectuées sur la parcelle. Nous conclurons sur l'importance de l'état initial vis-à-vis du comportement du sol au labour, sans établir la relation entre états successifs, ni discuter de la nature des processus de transformation au cours de l'opération.

\section{MATÉRIELS ET MÉTHODES}

\section{Conditions expérimentales}

L'expérimentation a été menée en région parisienne sur une parcelle $(85 \times 16,20 \mathrm{~m})$ du domaine expérimental INRAINA-PG de Grignon (78), sur un luvisol orthique (FAO) avec un horizon Ap de texture limonoargileuse (tableau I).

\section{Définition des traitements}

L'expérience des observations au champ montre qu'au sein d'une parcelle donnée, on est susceptible de voir coexister différents états structuraux à un moment donné (Manichon, 1988) :

- des états fragmentaires, avec des mottes de faible calibre $(<10 \mathrm{~cm})$, associées à une quantité importante d'éléments plus petits (terre fine) plus ou moins nettement individualisés;

- des états fragmentaires constitués de mottes de plus gros calibre $(>10 \mathrm{~cm}$ ) entre lesquelles les cavités ne sont pas comblées par de la terre fine;

- des états continus, plus ou moins tassés.

Ces états correspondraient respectivement aux zones qui n'avaient pas subi récemment de compactages, à celles qui avaient été compactées avant le dernier labour effectué, à celles enfin qui avaient subi après ce labour un certain compactage (opérations d'implantation ou d'entretien de la culture).

Nous avons vérifié que ces différents états existaient bien dans la parcelle choisie pour l'expérimentation. Nous les avons repérés. Nous avons décidé :

- de définir le premier traitement (NT) comme exempt de compactage supplémentaire avant labour; l'état initial présente une assez grande variabilité spatiale (caractérisée lors des observations), qui résulte de l'histoire culturale de la parcelle;

- d'augmenter la gamme de conditions initiales, en renforçant le tassement du sol à certains endroits; le deuxième traitement $(T)$ se définit alors par des compactages à deux dates différentes avant labour : 1 mois avant labour (T1); une semaine avant labour (T2); la distinction entre T1 et T2 vise à créer au moment du labour des états de même densité, mais de propriétés mécaniques différentes, résultant d'un phénomène de age-hardening, de l'effet d'une alternance humectation-dessication entre compactage et labour, ou d'une différence d'humidité au moment du compactage.

Les compactages réalisés avec le même tracteur ont été effectués de façon à obtenir un tassement aussi homogène que possible dans la parcelle concernée. Une opération de nivellement (herse rota-

Tableau I. Caractéristiques de la parcelle expérimentale.

\begin{tabular}{|c|c|c|c|c|c|c|c|}
\hline Antéprécédent & Précédent & $\begin{array}{l}t^{t} \quad A \\
<2 \mu(\%)\end{array}$ & $\begin{array}{c}1 \\
2-20 \mu(\%)\end{array}$ & $\frac{L}{20-50 \mu(\%)}$ & $\stackrel{s}{50-200 \mu(\%)}$ & $\begin{array}{c}S \\
200-2000 \mu(\%)\end{array}$ & $\begin{array}{l}\text { Matières organiques } \\
(\%)\end{array}$ \\
\hline $\begin{array}{l}\text { Luzerne } \\
1 \text { an }\end{array}$ & Blé & 25,9 & 23,2 & 34,9 & 12,1 & 3,9 & 2,1 \\
\hline
\end{tabular}


tive réglée très superficiellement) a été réalisée ensuite pour éliminer les bourrelets résiduels entre passages successifs. II en résulte un horizon superficiel composé principalement de petites mottes.

La comparaison des profils pénétrométriques moyens de $\mathrm{T} 1$ et $\mathrm{T} 2$ la veille du labour (fig 1) montre une nette différence de résistance à la pénétration pour une humidité et une densité équivalentes (tableau II). Par ailleurs, les variations de l'humidité de la couche labourée dans le délai de temps compris entre les deux dates de compactage sont susceptibles d'avoir entraîné une fissuration de l'état compacté sur T1.

\section{Conditions du labour}

La charrue utilisée est une charrue Huard, munie de deux corps 14 pouces à versoir universel avec un talon sur le corps arrière, d'une roue de jauge, sans rasettes, trainée par un tracteur 4 roues motrices Massey-Fergusson MF 595 de puissance maximale 88 Ch.

La profondeur du labour a été déterminée, de façon à travailler dans chaque traitement une masse de terre humide équivalente. Cette précaution expérimentale était nécessaire pour que les variations introduites dans les relations contrainte-déformations au moment du labour soient dues au seul état structural.

La vitesse d'avancement de la charrue a été enregistrée en continu à l'aide d'un radar à effet Dopler fixé sur le tracteur avec un angle défini par rapport au sol.

Les conditions au labour sont présentées au tableau II. Trois gammes de vitesses ont été explorées dans chaque traitement : lente, moyenne et rapide. II ne nous a pas été possible d'obtenir dans $T 1$, pour les gammes lente et rapide de cet essai, des valeurs de la vitesse identiques à NT et T2. La résistance à l'avancement du tracteur était en effet trop forte dans T1. Nous pouvons considérer, par contre, que les humidités de la couche à labourer et les masses de terre

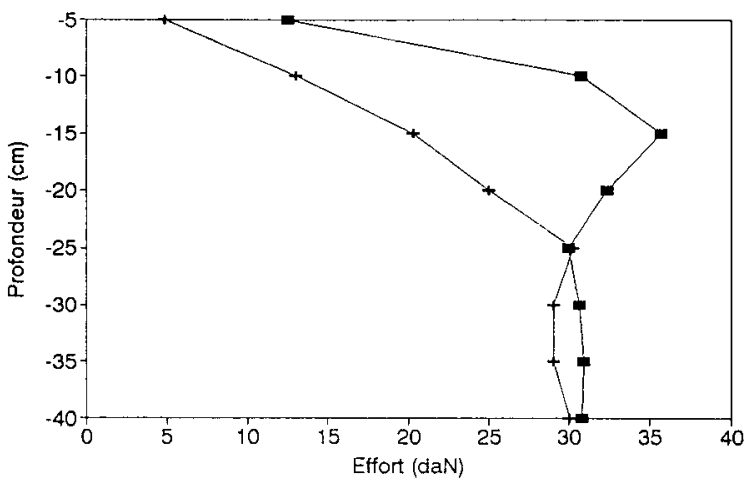

Fig 1. Profils de résistance pénétrométrique sur T1 et T2 la veille du labour. - - moyenne sur $\mathrm{T} 1$; - - - moyenne sur T2. T1 : continu et compact ancien, $W_{\text {comp }}=26 \%$; T2 : continue et compact récent, $W_{\text {comp }}=23 \%$. travaillées sont sensiblement identiques entre traitements.

\section{Dispositif}

La position des traitements a été choisie en fonction de transects pénétrométriques. Ceux-ci ont révélé la présence d'une zone plus résistante que le reste de la parcelle. Nous y avons affecté T1 pour s'assurer d'une différence d'état avec NT et d'une différence de propriétés mécaniques avec T2. II en résulte que les surfaces des différents traitements sont différentes. Hors zone de circulation, elles sont de $347,2 \mathrm{~m}^{2}(5,6 \times 62$ m) pour NT; 173,6 $\mathrm{m}^{2}(2,8 \times 62 \mathrm{~m})$ pour T1; 86,8 $\mathrm{m}^{2}$ $(2,8 \times 31 \mathrm{~m})$ pour T2. La forte surface affectée à NT est justifiée par la plus grande variabilité spatiale de l'état structural du sol attendue sur ce traitement. Elle permet d'y effectuer un nombre de contrôles plus importants que sur $\mathrm{T}$, où l'état initial est supposé plus homogène.

Les trois gammes de vitesses ont été pratiquées sur une même enrayure pour chaque passage de chaque traitement avec deux répétitions, sauf pour T2.

Les parcelles élémentaires correspondant aux trois gammes de vitesses (fig 2) ont des longueurs différentes pour que les enregistrements de vitesse aient lieu sur une même durée et puissent ainsi être valablement comparés.

\section{Caractérisation de l'état structural du sol}

La caractérisation de l'état structural du sol est fondée sur l'observation morphologique et stratifiée du profil cultural (Manichon, 1982a, 1987) avec une quantification de la surface des éléments structuraux et des cavités qui apparaissent sur une face verticale du profil perpendiculaire à la direction du labour (Caneill et al, 1982). Cette caractérisation en deux dimensions introduit un biais dans la représentativité des données visà-vis du volume que l'on cherche à scruter. Le biais in-
Répétition 2

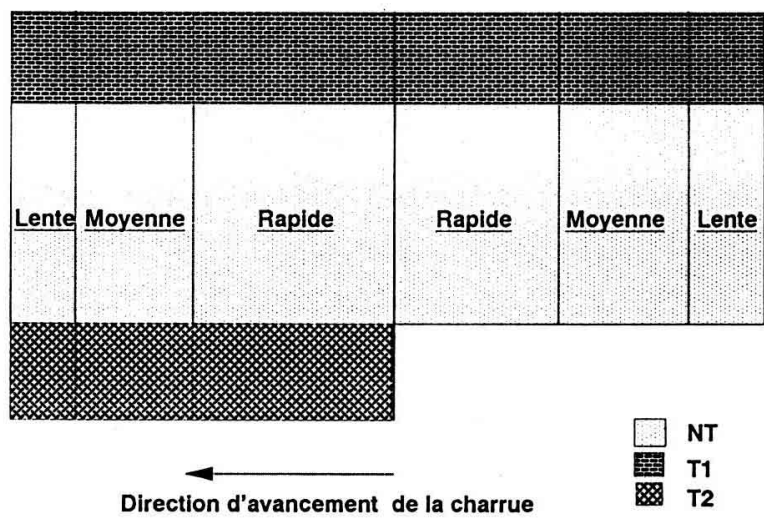

Fig 2. Dispositif. 
Tableau II. Conditions expérimentales.

\begin{tabular}{|c|c|c|c|}
\hline Traitement & $T 1$ & $T 2$ & $N T$ \\
\hline $\begin{array}{l}\text { Humidité moyenne de la couche labourée } \\
\text { au compactage }(\%)\end{array}$ & 26,0 & 23,0 & - \\
\hline $\begin{array}{l}\text { Humidité moyenne de la couche labourée } \\
\text { entre les deux compactages (\%) }\end{array}$ & $\begin{array}{l}0-5 \mathrm{~cm}: 5 \text { à } 25 \\
5-25 \mathrm{~cm}: 20 \text { à } 23\end{array}$ & $\begin{array}{l}7 \text { à } 28 \\
16 \text { à } 24\end{array}$ & $\begin{array}{r}7 \text { à } 27 \\
16 \text { à } 25\end{array}$ \\
\hline $\begin{array}{l}\text { Densité sèche } \\
\text { avant labour }\end{array}$ & $\begin{array}{l}0-5 \mathrm{~cm}: 1,3 \\
5-25 \mathrm{~cm}: 1,5\end{array}$ & $\begin{array}{l}0,9 \\
1,5\end{array}$ & $\begin{array}{l}1,2 \\
1,3\end{array}$ \\
\hline $\begin{array}{l}\text { Humidité moyenne de la couche labourée } \\
\text { au labour }(\%)\end{array}$ & 20,0 & 20,5 & 21,0 \\
\hline $\begin{array}{l}\text { Profondeur de labour } \\
(\mathrm{cm})\end{array}$ & 26,1 & 25,3 & 30,1 \\
\hline \multicolumn{4}{|l|}{ Vitesse de labour $(\mathrm{km} / \mathrm{h})$} \\
\hline Lente $(I)$ & 2,0 & 2,5 & 2,7 \\
\hline Moyenne (m) & 4,5 & 4,4 & 4,8 \\
\hline Rapide (r) & 5,6 & 6,7 & 6,7 \\
\hline $\begin{array}{l}\text { Masse de terre } \\
\text { travaillée }(\mathrm{kg} / \mathrm{m})\end{array}$ & 343 & 328 & 316 \\
\hline
\end{tabular}

troduit sur les distributions de taille d'objets sphériques par une caractérisation dans un espace à deux dimensions d'un système en trois dimensions est plus important lorsqu'il s'agit d'une distribution en nombre d'objets que lorsqu'il s'agit d'une distribution en mesure (longueur, surface, volume) (Coster et Chermant, 1989). Des travaux de simulation effectués à la station de science du sol INRA de Montfavet (Renault, non publié) sur des sphère sectionnées de manière aléatoire, de distribution volumique log-normale d'espérance 1 , bornées supérieurement, montrent que l'erreur sur le rayon moyen n'excède pas $13 \%$ lorsqu'on fait varier l'écart type de 0,5 à 1,5 pour des valeurs de bornes supérieures de 10 ou 50 . Cette erreur diminue si l'on tronque la distribution à 1 (mottes $<1 \mathrm{~cm}$ non identifiées) car les rayons les plus déformés sont de petite taille. Nous postulerons donc que les données obtenues sont représentatives du volume de sol avoisinant.

La procédure (Coulomb, 1991) comporte :

- une préparation du profil au couteau et au soufflet faisant ressortir les éléments structuraux et cavités et permettant de noter l'état interne des premiers;

- une photographie du profil avec un objectif peu déformant, un réglage fixe de la profondeur de champ, des prises de vues rapprochées (tous les $6,5 \mathrm{~cm}$ ) parallèles au plan du profil; l'échelle des photos est le 15/ 100 ;

- une interprétation stéréoscopique de la partie centrale de la photo produisant un document simple où ne figurent que les contours des éléments structuraux et de la bande de labour, avec une épaisseur de trait à peu près constante $(0,7 \mathrm{~mm})$;

- un traitement informatique du document composé d'une acquisition standardisée de l'image (position du document dans un plan perpendiculaire à l'axe optique de la caméra, ouverture de l'objectif, éclairage, gain, offset) par une caméra video $C C D$ associée à une carte $512 \times 512$ pixels numérisant en 256 niveaux de gris, d'une binarisation au seuil de 250 (tous les pixels d'intensité supérieure à 250 deviennent blancs, les autres sont noirs), d'une détection des contours fournissant pour chaque objet identifié, son périmètre, sa surface, sa plus grande longueur, sa plus petite longueur, ses coordonnées barycentriques.

À chaque étape, on trouve des sources d'erreur dépendantes de la taille des objets (résolution graphique, tracé des contours lors de la préparation du document, détection des contours). D'autres sources d'erreur sont plus localisées et liées au relief donné au profil, aux paramètres de prise de vue, à la complexité des formes. Les erreurs intervenant lors de la préparation de la face d'observation sont difficilement quantifiables car cette préparation relève d'un savoirfaire d'expert. L'unicité de l'expert rend néanmoins plausible l'unicité du biais introduit par tous les traitements. Une estimation des autres erreurs à partir d'objets de taille et formes connues a montré que la chaîne de traitement après préparation du profil conduisait à une précision sur les surfaces de l'ordre 
de $\pm 5 \%$ mais qu'une sous-estimation est à craindre pour les petits objets correspondant à des mottes de moins de $5 \mathrm{~cm}$ de longueur. Celle-ci peut atteindre $13 \%$.

Nous distinguons ainsi les mottes de plus de $2 \mathrm{~cm}$ selon leur état interne et leur calibre (plus grande longueur de la motte), la terre fine, les vides de plus de 1 $\mathrm{cm}$. Les états internes sont repérés sur le terrain. Ils sont présentés dans le tableau III. Comme nous nous intéressions à une opération de fragmentation nous avons distingué, au sein de l'état $ø$ deux niveaux de fissuration.

Nous avons effectué nos observations juste après labour en observant une section travaillée correspondant à la largeur travaillée par passage de charrue, dans chaque situation*. L'unité expérimentale retenue correspond donc à deux bandes de labour (fig 3). Pour caractériser les traitements avant le labour, nous avons adopté une méthode analogue. L'unité expérimentale a été choisie dans ce cas de manière à permettre la confrontation entre les variabilités avant et après labour. Elle correspond à une section labourable, soit $75 \mathrm{~cm}$ de large multiplié par la profondeur de la couche labourée.

Pour chaque unité, les surfaces individuelles des mottes correspondant à un même type d'objet (mottes de même état interne, de même calibre, terre fine, vides) ont été cumulées dans l'analyse qui suit.

\section{Détermination des variables représentatives de l'effet direct du labour}

L'état du sol à une certaine date après labour est le résultat du labour lui-même, dont on veut caractériser l'effet, mais aussi de l'évolution de l'état créé par le la- bour, entre ce dernier et la date d'observation, sous l'action des facteurs climatiques.

Nous avons donc cherché à réduire au maximum la durée de la période d'observation sur le terrain. Celleci est de 14 jours. Mais nous avons aussi adopté une stratégie de dates d'observation nous permettant de détecter les variables les plus sensibles à une évolution sous l'action du climat, pour ne retenir dans l'analyse que celles qui en sont les plus indépendantes. Nous avons ainsi répété les observations en début et en fin de période dans le traitement NT, où la gamme de variation de l'état structural est a priori plus importante et plus à même de révéler des évolutions.

II est difficile de distinguer les mottes $\Gamma$ et la terre fine sur le terrain quand la taille des éléments structuraux avoisine $1 \mathrm{~cm}$. La sensibilité de la terre fine à une reprise en masse sous l'action des pluies est, par ailleurs, forte. Les variables susceptibles d'être les plus sensibles à la date d'observation sont donc la surface en mottes $\Gamma$ et la surface en terre fine.

Pour tester cette hypothèse, nous avons utilisé le test non paramétrique de Kruskal-Wallis sur les 48 unités de NT observées : test des rangs appliqué à la comparaison de plusieurs échantillons indépendants (Dagnelie, 1975). On teste, grâce aux répétitions des

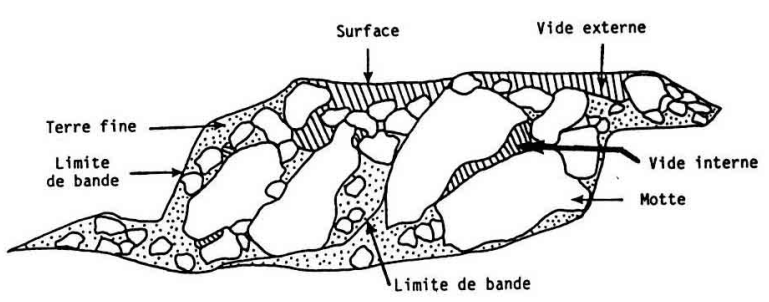

Fig 3. Définition de l'unité expérimentale.

Tableau III. Caractéristiques des états internes distingués (Manichon, 1982a).

\section{État interne}

$\varnothing$

$\Gamma$

Terre fine

\section{Principales caractéristiques}

Aspect continu • Faces de fragmentation peu rugueuses de forme conchoïdale - Porosité visible très faible ou nulle • Cohésion à sec élevée

État delta avec amorces de fissures sous l'action d'une fragmentation

Agrégats de morphologie variable discernables dans les mottes • Rugosité importante après fragmentation •

Porosité structurale non nulle $\cdot$ Cohésion plus faible

Agrégats individualisés de diamètre $<1 \mathrm{~cm}$

\footnotetext{
" On appelle situation la combinaison "traitement $x$ vitesse $x$ répétition" codée par le nom du traitement, l'initiale de la vitesse correspondante ( $1, \mathrm{~m}$ ou $r$ ) et le numéro de la répétition (pour NT et $\mathrm{T} 1$ ).
} 
observations, la stabilité dans le temps de chacune des variables. Seules la surface en mottes $\Gamma$ et la surface en terre fine varient significativement au niveau $\alpha$ $=5 \%$. Exprimées en pourcentage de la surface de l'unité, la surface en mottes $\Gamma$ passe de $15 \%$ en début des observations à $30 \%$ à la fin de celles-ci, alors que la surface en terre fine passe de 70 à $55 \%$ (valeurs moyennes de chaque date d'observation). En revanche, le cumul des deux variables ne varie pas significativement. L'hypothèse d'un passage progressif de l'état de terre fine à l'état de mottes $\Gamma$, sous l'action des agents climatiques est donc admissible. Nous ne ferons plus par conséquent la distinction entre mottes $\Gamma$ et terre fine dans la suite de notre analyse.

La liste des variables retenues pour l'analyse est présentée dans le tableau IV. Nous n'avons pas distingué de classes au-delà de $20 \mathrm{~cm}$ pour caractériser le calibre des mottes $\Delta$ dans NT, T1 et T2 car les mottes de plus de $30 \mathrm{~cm}$ sont absentes dans NT avant labour (fig 4). Après labour, nous comparerons les proportions des mottes de plus de $30 \mathrm{~cm}$ dans $\mathrm{T} 1$ et dans T2.

Nous avons analysé la variabilité de l'état structural du sol après labour sur la base d'une analyse statistique descriptive des variables retenues, l'analyse factorielle des correspondances (AFC). L'objectif de cette analyse est de déterminer, sans hiérarchie préalable, les variables ou combinaisons de variables les plus représentatives de la variabilité observée à partir desquelles nous pouvons caractériser celle-ci. L'AFC a été effectuée sur les surfaces mesurées de chaque type morphologique. Nous exprimerons les résultats sur les graphiques en pourcentage de la surface de l'unité pour les différents états internes. En revanche, pour rendre la description des calibres des mottes $\Delta$ et $\varnothing$ indépendante de l'importance surfacique de ces types de mottes dans l'unité, les surfaces cumulées des mottes $\Delta$ et $\emptyset$ d'un calibre donné seront exprimées par rapport à la surface totale de mottes $\Delta$ et $\varnothing$ (mottes $ø 1$ et $ø 2)$. Nous préciserons $\%(\mathrm{~S} \Delta+\mathrm{S} ø)$ dans les cas concernés.

Tableau IV. Variables retenues pour l'analyse des états structuraux des sols avant et après labour.

\footnotetext{
SItf Surface des mottes $\Gamma$ et terre fine

$\mathrm{S} \Delta \quad$ Surface des mottes $\Delta$

Sø1 Surface des mottes $ø$ à 1 niveau

Sø2 Surface des mottes $ø$ à 2 niveaux

SVI Surface des vides internes à la bande de labour sans contact avec la surface

SVS Surface des vides en contact avec la surface

$\mathrm{S}_{2-5}$ Surface des mottes de calibre $<5 \mathrm{~cm}$

$S_{5-10}$ Surface des mottes de calibre entre 5 et $10 \mathrm{~cm}$

$S_{10-15}$ Surface des mottes de calibre entre 10 et $15 \mathrm{~cm}$

$S_{15-20}$ Surface des mottes de calibre entre 15 et $20 \mathrm{~cm}$

$\mathrm{S}_{>20}$ Surface des mottes de calibre $>20 \mathrm{~cm}$
}

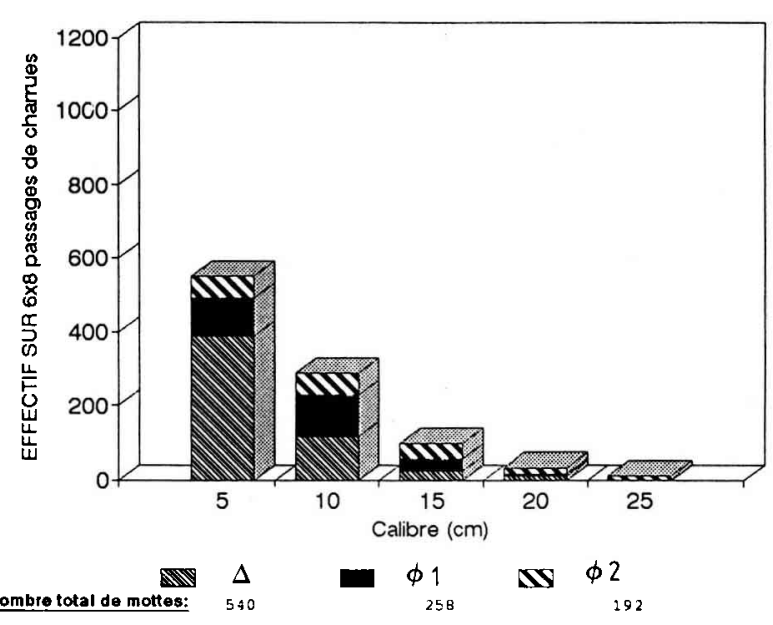

Fig 4. Distribution des calibres avant labour sur NT.

\section{RÉSULTATS : ANALYSE DES ÉTATS AVANT ET APRÈS LABOUR}

Rappelons que l'unité expérimentale est la section correspondant à un passage de charrue, c'est-à-dire à deux bandes de labour. Étant donné le plan de sondage adopté, le nombre d'unités observées est de 27 (19 pour NT, 4 pour $\mathrm{T} 1,4$ pour T2) avant labour, et 84 après labour (48 pour NT, 24 pour T1, 12 pour T2).

\section{Analyse de la variabilité de l'état structural du sol avant labour : caractérisation des traitements}

\section{Variabilité des états internes des éléments structuraux}

Les résultats (tableau $V$ ) montrent qu'il existe une disjonction complète entre les valeurs de $\mathrm{S} \Gamma \mathrm{tf}$ (en pourcentage de la surface de l'unité) des unités de NT d'une part, et celles de T1 et T2 d'autre part. NT se caractérise par une forte proportion de mottes $\Gamma$ et de terre fine et une faible proportion de mottes $\Delta$ et $\varnothing$.

$T 1$ et $T 2$, à forte teneur en mottes $\Delta$ et $\varnothing$, se distinguent entre eux par l'état de fissuration de ces mottes. Celui-ci est plus développé sur T1 que sur T2. Sø2 reste néanmoins inférieur à $5 \%$ sur $\mathrm{T} 1$, signe d'une fissuration encore peu prononcée. La forte variabilité de $S \Delta$ et $S \varnothing 1$ dans T2 s'explique par l'amorce d'une très légère fissuration sur une unité de T2 et l'absence de fissuration sur les autres. 
Tableau V. Caractérisation des états internes des éléments structuraux du sol sur les traitements avant labour.

\begin{tabular}{lccccc}
\hline \multicolumn{5}{c}{ Teneur (\%) : moyenne (min-max) } \\
\cline { 2 - 6 } Traitement & STIf & S $\Delta$ & Sø1 & Sø2 & S $\Delta+$ Sø1+Sø2 \\
\hline T1 $(n=4)$ & $23(20-26)$ & $33(17-48)$ & $38(21-52)$ & $2(1-3)$ & $73(70-81)$ \\
T2 $(n=4)$ & $18(10-25)$ & $63(8-85)$ & $16(0-64)$ & $0(0-0)$ & $79(72-86)$ \\
NT $(n=19)$ & $79(59-91)$ & $5(0-17)$ & $6(0-13)$ & $7(1-18)$ & $18(9-37)$ \\
\hline
\end{tabular}

\section{Variabilité des calibres des mottes}

T1 et T2 se caractérisent par l'importance des mottes de calibre supérieur à $20 \mathrm{~cm}$ (essentiellement supérieurs à $30 \mathrm{~cm}$ ). Celles-ci représentent en effet plus de $88 \%$ des mottes $\Delta$ et $ø$ (tableau VI). La présence de ces mottes est le résultat d'un tassement pas totalement homogène de I'horizon par les opérations de compactage. Les calibres sont plus faibles dans NT avec une forte variabilité entre unités. Dix unités (sur 19) possèdent des mottes supérieures à $15 \mathrm{~cm}$. Ces mottes ne représentent néanmoins jamais plus de $30 \%$ de la surface de l'unité.

\section{Conclusion sur l'état avant labour}

Les compactages exercés sur la parcelle ont conduit à une différenciation des états structuraux (état interne et taille des mottes) dont la variabilité se structure selon les traitements définis. La variabilité intratraitement est faible dans $\mathrm{T} 1$ et dans T2. Elle est plus forte dans NT en raison de la présence locale de mottes supérieures à 15 $\mathrm{cm}$ dont l'importance surfacique reste modérée à l'échelle de l'unité. Environ la moitié des unités de NT sont ainsi concernées par la présence de ces mottes.

\section{Analyse de la variabilité de l'état structural du sol après labour}

\section{Variabilité des états internes des mottes}

Les deux premiers axes fournis par l'AFC sur les variables indicatrices de l'état interne des éléments structuraux et les 84 unités observées expliquent $78,3 \%$ de la variabilité totale. Ils apportent l'essentiel de l'information.

Le premier axe oppose les variables STtf aux variables $S \Delta$ et $S ø 1$. Le deuxième axe est caractérisé par les variables $S ø 1$ et $S \Delta$ de signes opposés sur l'axe. Ces trois variables permettent de décrire l'essentiel de la variabilité observée. La somme des variables impliquées dans I'AFC est égale à la surface de l'unité. Nous avons donc représenté sur un triangle les proportions de $S \Gamma \mathrm{tf}, \mathrm{S} \Delta$ et des surfaces occupées par les autres types d'états internes. Pour souligner la présence de vides, nous avons identifié les unités où la variable VI est non nulle (fig 5). La surface totale des vides demeurant faible, le troisième axe du triangle est représentatif de Sø1+ Sø2, c'est-à-dire de la surface occupée par les mottes $\varnothing$ (notée $\mathrm{S} \varnothing$ ).

On constate que le traitement NT a une forte teneur en mottes $\Gamma$ et en terre fine alors que le traitement $T$ ( $T 1$ et $T 2)$ a une forte teneur en

Tableau VI. Caractérisation des calibres des mottes sur les traitements avant labour.

Teneur \% (SA+Sø) : moyenne (min-max)

\begin{tabular}{lccccc} 
Traitement & $S_{2-5}$ & $S_{5-10}$ & $S_{10-15}$ & $S_{15-20}$ & $S_{>20}$ \\
\hline T1 $(n=4)$ & $4(3-5)$ & $4(3-6)$ & $1(0-3)$ & $0(0-0)$ & $91(89-92)$ \\
T2 $(n=4)$ & $4(3-6)$ & $2(1-5)$ & $2(0-5)$ & $0(0-0)$ & $91(88-94)$ \\
NT $(n=19)$ & $19(2-48)$ & $34(7-86)$ & $25(0-44)$ & $11(0-49)$ & $11(0-78)$
\end{tabular}




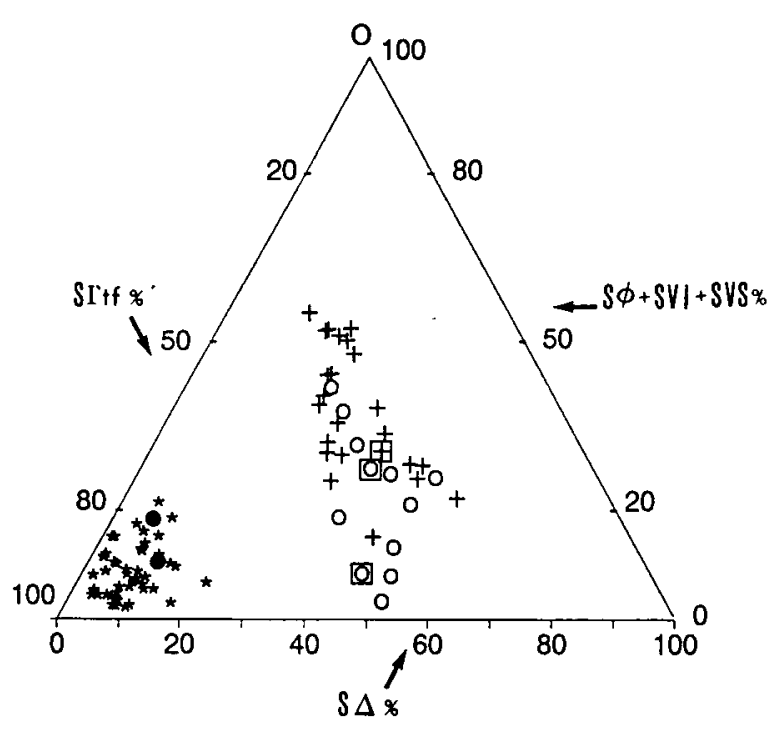

Fig 5. Teneurs surfaciques, par rapport à la surface de l'unité, des éléments structuraux selon leur état interne après labour. * : NT (fragmentaire) avec SVI égal à 0; : NT (fragmentaire) avec SVI non nul; + : T1 (continu et compact ancien); $0:$ T2 (continu et compact récent). Les croix et cercles encadrés correspondent aux unités de $\mathrm{T} 1$ et $\mathrm{T} 2$, où Vl est nul. Les coordonnées sur chaque axe s'obtiennent par projection parallèlement au triangle (flèches).

mottes $\Delta$. Seules les unités de T1 et T2 sont associées à des teneurs surfaciques en vides internes supérieures à $1 \%$. Le traitement $T$ se caractérise par une variabilité de $\mathrm{S} \varnothing=(\mathrm{S} \varnothing 1+\mathrm{S} ø 2)$ plus importante qu'avant labour. Les unités de T2 restent plutôt moins fissurées que celles de $\mathrm{T} 1$. En effet, Sø2 représente en moyenne $14 \%$ dans T1 contre $4 \%$ dans T2 avec une différence significative au niveau $1 \%$ (test $t$ de student).

\section{Variabilité des calibres des mottes}

L'AFC sur les surfaces occupées par les mottes $\Delta$ et $\varnothing$ dans l'unité, selon leur calibre, fournit deux axes décrivant $75,4 \%$ de la variabilité.

L'axe 1 est caractérisé par $S_{>20}$. L'axe 2 est caractérisé par S15-20 de signe opposé aux variables indicatrices des faibles calibres. Nous retenons donc une représentation triangulaire des trois variables $S_{>20}, S_{15-20}, S_{2-5}+S_{5-10}+S_{10-15}$ (fig 6).

Les unités avec les plus faibles calibres $(100 \%$ de mottes $<15 \mathrm{~cm}$ ) sont toutes des unités de NT. Comme avant labour, certaines unités de NT ont néanmoins des mottes de calibre supérieur à

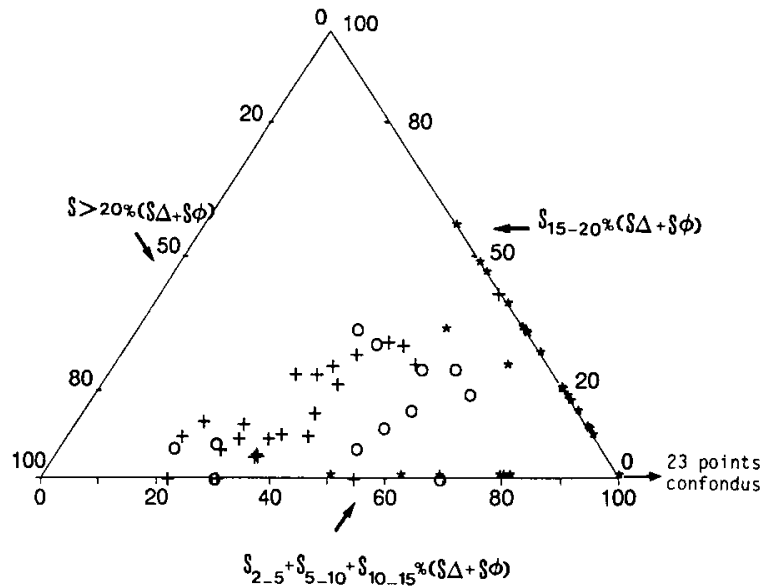

Fig 6. Teneurs surfaciques, par rapport à la surface totale des mottes $\Delta$ et $\sigma$, selon leur calibre après labour. * : NT (fragmentaire); + : T1 (continu et compact ancien); $0:$ T2 (continu et compact récent). Les coordonnées sur chaque axe s'obtiennent par projection parallèlement au triangle (flèches).

$15 \mathrm{~cm}$ qui peuvent représenter jusqu'à $50 \%$ de la surface des mottes*. Nous constatons qu'environ la moitié des unités de NT est concernée par la présence de ces mottes supérieures à $15 \mathrm{~cm}$. La taille des mottes est plus importante dans $T$ où les valeurs de $S_{>20}$ sont les plus fortes avec une forte variabilité entre unités. La teneur surfacique en mottes de plus de $20 \mathrm{~cm}$ est en moyenne plus forte dans $\mathrm{T} 1$ que dans $\mathrm{T} 2$ ( $48 \%$ dans $\mathrm{T} 1$ contre $38 \%$ dans $\mathrm{T} 2$ ). La différence est significative au niveau $7 \%$ (test $t$ de student).

Nous avons cherché si la variabilité des calibres de mottes dans le traitement tassé se structurait selon $\mathrm{T} 1$ et $\mathrm{T} 2$ et selon les vitesses de labour pratiquées. Les résultats (tableau VII) montrent que les calibres sont plus importants dans $\mathrm{T} 1$ que dans $\mathrm{T} 2$, mais que la distinction porte sur les mottes de plus de $30 \mathrm{~cm}$. Moins de $10 \%$ de la surface occupée par des mottes $\Delta$ et $\varnothing$ l'est par des mottes de plus de $30 \mathrm{~cm}$ dans $\mathrm{T} 2$, contre plus de $10 \%$ dans T1. La différence entre les situations labourées à des vitesses différentes est faible. II semblerait que les mottes soient plus petites lorsque la vitesse est plus lente, ce qui est contraire à ce que nous prévoyions.

\section{Conclusion sur l'état après labour}

Ces analyses montrent que la variabilité des états structuraux après labour porte sur la sur-

* La surface des mottes est faible par rapport à celle de l'unité dans les cas concernés. 
Tableau VII. Calibres des mottes après labour sur les traitements tassés pour des situations labourées à des vitesses différentes.

\begin{tabular}{lcc}
$\begin{array}{l}\text { Situation } \\
\text { (moyenne }\end{array}$ & $\begin{array}{c}\text { Teneur surfacique } \\
\text { des mottes de calibre }\end{array}$ & $\begin{array}{c}\text { Teneur surfacique } \\
\text { des mottes }\end{array}$ \\
$\begin{array}{cc}\text { de unités) } \\
\text { supérieur à } 20 \mathrm{~cm}\end{array}$ & $\begin{array}{c}\text { supérieur à } 30 \mathrm{~cm} \\
\%(S \Delta+S \sigma)\end{array}$ \\
& $\%(S \Delta+S \sigma)$ \\
\hline
\end{tabular}

$\begin{array}{lll}\text { T2l2 } & 26 & 4 \\ \text { T2m2 } & 40 & 8 \\ \text { T2r2 } & 49 & 6 \\ & & \\ \text { T1l1 } & 40 & 11 \\ \text { T1l2 } & 51 & 10 \\ \text { T1m1 } & 39 & 23 \\ \text { T1m2 } & 64 & 29 \\ \text { T1r1 } & 58 & 15 \\ \text { T1r2 } & 44 & 20\end{array}$

face occupée dans le profil par les mottes $\Delta$ et $\varnothing$, l'état de fissuration et le calibre des mottes. Elle est structurée selon les traitements après labour.

NT se distingue nettement de $T$ par une teneur surfacique plus faible en mottes $\Delta$ et $\varnothing$. Dans NT, les mottes sont de plus faible calibre. On note aussi l'absence de vides supracentimétriques.

Le traitement $T$ est marqué par une forte variabilité des calibres et de l'état de fissuration des mottes. Les mottes les plus fissurées occupent une surface en moyenne plus importante sur T1 que sur T2.

Les variabilités de l'état de fissuration et du calibre des mottes ne peuvent être interprétées indépendamment l'une de l'autre et doivent être reliées aux processus de fragmentation dont l'analyse n'est pas l'objet du présent article.

\section{DISCUSSION ET CONCLUSION}

L'analyse de la variabilité de l'état structural de la couche labourée avant labour montre que les traitements appliqués ont effectivement conduit à des états différenciés. II y a disjonction entre le traitement tassé (T1 et T2) et NT. Celle-ci porte sur la teneur surfacique en mottes $\Gamma$ et terre fine, la teneur en mottes $\Delta$ et $\varnothing$, et la teneur surfacique en mottes de plus de $20 \mathrm{~cm}$. Enfin, NT se caractérise par une variabilité importante de la taille des mottes. Par ailleurs, T1 a tendance à être plus fissuré que T2. Après labour, la disjonction observée entre $T$ et NT et la tendance à la fissuration plus développée sur $\mathrm{T} 1$ se retrouvent sur les mêmes variables. La variabilité de taille des mottes dans NT reste de même ampleur qu'avant labour. Cela confirme le poids déterminant de l'état initial sur l'état transformé par le labour.

Dans les conditions de texture et teneur en eau explorées, les conséquences de l'état avant labour sur l'état après labour sont les suivantes :

- lorsque l'état est fragmentaire avant labour, l'état se caractérise après labour par des mottes de faible calibre, une faible surface en mottes tassées, l'absence de vides supracentimétriques;

- lorsque l'état est continu et compact avant labour, l'état après labour se caractérise par la présence de mottes compactes de fort calibre; la taille et l'état de fissuration des mottes varient alors selon l'ancienneté du compactage. Après labour, les calibres sont plus importants et l'état de fissuration est plus développé là où le compactage était le plus précoce. Ce résultat est une conséquence de la différence de résistance ou d'état de fissuration initial entre les deux types d'état compacté, dont l'origine pourrait être liée à la différence d'histoire climatique entre compactage et labour, ou bien à la différence de teneur en eau au compactage. Celle-ci, plus importante dans T1, pourrait avoir induit une plus grande résistance mécanique des mottes sans variation de densité, et une fissuration plus importante en raison d'histoires hydriques différentes entre $\mathrm{T1}$ et T2.

L'effet de la vitesse de labour sur l'état structural du sol après labour est, par contre, assez faible. II ne concerne que le calibre des mottes et semble contraire à celui attendu d'après les données bibliographiques existantes. En effet, pour $\mathrm{T} 2$ et T1, les profils présentant les calibres les plus faibles correspondent aux labours les plus lents, alors que certains auteurs mentionnent l'effet inverse (Hénin et al, 1969; Faure, 1970; Dalleine, 1978).

Une variabilité importante de l'état de fissuration et du calibre des mottes est apparue après labour dans le traitement tassé. Nous montrons qu'elle se structure pour partie selon les modalités du traitement tassé et ne peut s'expliquer par la vitesse de labour. On peut penser qu'elle est liée à une variabilité de l'état initial du sol, non repérée dans le traitement compacté avant labour, dont les effets sur les processus de fragmentation seraient non négligeables. 
La discussion précédente montre que l'état structural initial de la parcelle n'est pas sans conséquences sur l'état transformé alors que les mesures de vitesse effectuées sur l'ensemble tracteur-charrue utilisé permettent d'affirmer que la gamme de conduites de l'outil explorée correspond à la gamme couverte au champ dans la pratique.

Cela traduit, sur la parcelle étudiée, l'impossibilité de modifier à volonté l'état structural du sol. II ne suffit pas de faire varier la vitesse de la charrue pour annuler un tassement. II paraît donc impossible d'éliminer des tassements liés à des interventions culturales antérieures en une seule opération de labour. La pratique d'un labour à une humidité plus fabile, voisine de la limite de retrait (teneur en eau de $9 \%$ sur la parcelle de Grignon) pourrait néanmoins induire une réduction plus forte de la taille des blocs compactés (Coulomb, 1991).

Les diagnostics au champ avaient permis à Manichon et Sebillotte (1975) de supposer la corrélation entre l'état du profil après labour et l'état avant labour, lui-même résultat de l'ensemble du passé cultural. Nous confirmons dans le cas du labour la dépendance entre l'évolution du profil cultural et son état avant intervention. II est donc indispensable de prendre en compte les effets cumulatifs des systèmes de culture pour gérer les conditions d'intervention du labour (Manichon, 1988; Coulomb et al, 1990). Pour cela, il est important de connaître la nature de la relation entre l'état initial du sol et son état transformé par le labour. Sur la parcelle étudiée, nous avons pu montrer que la variabilité des états avant labour était plus faible intratraitement qu'intertraitement, ce qui nous autorise à y établir la relation état initial - état transformé sur des unités de dimension supérieure au passage de charrue.

Grâce à la caractérisation morphologique adoptée, des perspectives de quantification de l'interdépendance entre les états avant et après labour s'ouvrent. La méthode de caractérisation utilisée a permis en effet de comparer sur une parcelle la variabilité des états structuraux du sol avant et après labour, sur la base de critères quantitatifs dont on peut analyser l'évolution. Ils sont indicateurs de la représentativité surfacique dans le profil des mottes d'un état interne donné, de leur calibre, et des espaces poraux macroscopiques. Cette méthode n'offre néanmoins qu'une vision en deux dimensions de la structure du sol de la couche labourée. Une quantification plus précise du biais introduit par rapport à l'estima- tion de teneurs volumiques serait donc souhaitable. Une simplification de la chaîne de traitement, visant à diminuer les sources d'erreurs possibles et diminuer le temps de traitement serait peut-être aussi à envisager en fonction des critères structuraux réellement utiles à la compréhension du fonctionnement du peuplement végétal et/ou de la dynamique de l'eau et des éléments minéraux dans la couche labourée.

\section{RÉFÉRENCES}

Atterberg (1911) Cité par Baver LD, Gardner WH, Gardner WR (1972) The Dynamic properties of Soils. In: Soils Physics. John Wiley and Sons, Inc, New York, 74-129

Caneill J, Coulomb I, Manichon H, Roger-Estrade J (1992) A quantitative method for describing soil structure. Proc 2nd Congr European Soc Agron, 338-339

Coster M, Chermant $J$ (1989) Précis d'analyse d'images. CNRS plus, CNRS, $545 p$

Coulomb I (1991) Analyse quantitative du comportement du sol au labour : rôle de l'état structural initial. Thèse INA-PG, Paris, $230 \mathrm{p} \mathrm{(+} \mathrm{annexes)}$

Coulomb l, Manichon H, Roger-Estrade J (1990) Évolution de l'état structural sous l'action des systèmes de culture. In: Actes du colloque "La structure des sols et son évolution» (Boiffin J, Marin-Laflèche A, eds). INRA Laon, 137-155

Dagnelie $P$ (1975) Théories et méthodes statistiques. Les Presses Agronomiques de Gembloux, $2^{\mathrm{e}}$ éd, vol 2, Gembloux, 390-392

Dalleine $E$ (1978) Les façons en travail du sol, tome II. Études du CNEEMA, $n^{\circ} 438$

Dantu $P$ (1957) Contribution à l'étude mécanique et géométrique des milieux pulvérulents. Proc of the 4th Int Conf on Soil Mechanics and Foundations Eng 1, Butterworth, Londres, 144-148

Dexter AR (1988) Strength of soil aggregates and of aggregate beds. In: Impact of water and external forces on soil structure (Drescher, Horn, De Boodt, eds). Catena sup 11, Catena Verlag D, Cremlingen Dested, 35-52

Faure A (1970) Contribution à l'étude de l'action d'un versoir sur le sol travaillé par un versoir de charrue. Ann Agron 21, 159-195

Faure A (1971) Contribution à l'étude du mécanisme du compactage des sols. Ann Agron 22, 487-520

Guérif $J$ (1988) Résistance en traction des agrégats terreux : influence de la texture, de la matière organique et de la teneur en eau. Agronomie 8, 379386

Guérif J (1990) Conséquences de l'état structural sur les propriétés et les comportements physiques et mécaniques. In: Actes du colloque "La structure 
des sols et son évolution" (Boiffin J, Marin-Laflèche A, eds). INRA Laon, 71-90

Hénin S, Feodoroff A, Gras R, Monnier G (1969) Amélioration du profil cultural. II. Le travail du sol. In: $L e$ profil cultural, $2^{\mathrm{e}}$ éd. Masson, Paris, 173-198

Manichon $\mathrm{H}$ (1982a) Influence des systèmes de culture sur le profil cultural : élaboration d'une méthode de diagnostic basée sur l'observation morphologique. Thèse Docteur Ingénieur INA-PG, Paris, $214 \mathrm{p}+$ (annexes)

Manichon H (1982b) L'action des outils sur le sol : appréciation de leurs effets par la méthode du profil cultural. Sci Sol Bull AFES 3

Manichon H (1987) Observation morphologique de l'état structural et mise en évidence d'effets de compactage des horizons travaillés. In: Soil compaction and regeneration (Monnier G, Gosse MJ, eds). Balkema, Rotterdam, 39-52

Manichon $H$ (1988) Compactage, décompactage du sol et systèmes de culture. CR Acad Agric Fr 74, 43-54

Manichon $H$, Sebillotte $M$ (1975) Analyse et prévision des conséquences des passages successifs d'outils sur le profil cultural. Bull Tech Inf 302/303, 569577

Manichon $H$, Roger-Estrade J, Leterme $P$ (1988) Analyse des profils culturaux et des itinéraires techniques. Conséquences pour le blé. Doc mult INA-
PG, Chaire d'agronomie. Convention INRAONIC, volet 2, $\mathrm{RCl}$

Monnier G, Guérif J (1988) Conséquences physiques et agronomiques du tassement des sols. CR Acad Agric Fr 74, 29-42

Nichols ML, Baver LD (1930) An interpretation of the physical properties of soil affecting tillage and implements design by means of the Atterberg consistency constants. Trans 2nd Int Congr Soil Sci 6, 175-188

Papy F (1984) Comportement du sol sous l'action des façons de reprise d'un labour au printemps. Effet des conditions climatiques et de l'état structural. Thèse Docteur Ingénieur INA-PG, Paris, 232 p (+ annexes)

Rémy JC (1971) Influence de la constitution physique des sols sur leur comportement mécanique; signification des limites d'Atterberg en matière de travail du sol. Ann Agron 22, 267-290

Stengel $P$ (1990) Caractérisation de l'état structural du sol. Objectif et méthodes. In: Actes du colloque «La structure des sols et son évolution" (Boiffin J, Marin-Laflèche A, eds). INRA Laon, 15-36

Travers T, Ami M, Bideau D, Gervois A, Lemaitre J, Messager JC, Troadec JP (1988) Compression de milieux granulaires. Modèles à deux dimensions. Rev Fr Géotechnique 43, 20-34

Utomo WH, Dexter AR (1981) Age hardening of agricultural top soils. J Soil Sci 32, 335-350 